\title{
深度特征融合的非刚性三维形状局部匹配
}

\author{
韩丽, 佟宇宁, 朴京钰, 徐圣斯, 王晓旻, 兰鹏燕, 于冰 \\ (辽宁师范大学计算机与信息技术学院 大连 116000) \\ (h1_dlls@dl.cn)
}

\begin{abstract}
摘 要: 针对海量、异构三维形状匹配与智能检索技术的需求, 提出了一种基于级联卷积神经网络(F-PointCNN)深度 特征融合的三维形状局部匹配方法. 首先, 采用特征袋模型, 提出几何图像表示方法, 该几何图像不仅能够有效区分 同类异构的非刚性三维模型, 而且能够揭示大尺度不完整三维模型的结构相似性. 其次, 构建级联卷积神经网络学 习框架 F-PointCNN, 其中, BoF-CNN 从几何图像中学习深度全局特征, 建立融合局部特征与全局特征的点特征表示; 进而对 Point-CNN 进行点特征的细化与提纯, 生成具有丰富信息的深度融合特征, 有效提高形状特征的区分性与鲁 棒性. 最终, 通过交叉矩阵度量方法高效实现非刚性三维模型的局部形状匹配. 在公开的非刚性三维模型数据库的 实验结果表明，该方法提取的特征在大尺度变换的形状分类及局部形状匹配中具有更强的识别力与更高的匹配精度.
\end{abstract}

关键词：局部匹配；特征融合; 卷积神经网络; 非刚性三维模型

中图法分类号: TP391.41 DOI: 10.3724/SP.J.1089.2021.18446

\section{Non Rigid 3D Shape Partial Matching Based on Deep Feature Fusion}

\author{
Han Li, Tong Yuning, Piao Jingyu, Xu Shengsi, Wang Xiaomin, Lan Pengyan, and Yu Bing
}

(Faculty of Computer and Information Technology, Liaoning Normal University, Dalian 116000)

\begin{abstract}
To meet the requirements of massive and heterogeneous 3D shape partial matching and intelligent retrieval technology, a 3D shape local matching method based on the deep fusion feature of F-PointCNN is proposed. First, the feature bag $(\mathrm{BoF})$ learning model is used to propose an geometric image representation, which can not only effectively distinguish heterogeneous non-rigid 3D models of the same kind, but also reveal the structural similarity of large-scale incomplete 3D models. Next, a cascaded convolutional neural network slearning framework (F-PointCNN) is constructed, where BoF-CNN learns the deep global feature from BoF geometric images and establishes the point feature representation that integrates the local feature and the global feature; Point-CNN refines the point feature and generates deep feature representation which effectively improves the discriminative ability and robustness. Finally, the local shape matching of non rigid 3D model is realized by cross matrix measurement. The open non-rigid 3D shape databases are used to carry out a series of experiments, the results show that the features extracted by proposed method have stronger discriminative ability in large-scale transformation shape classification and higher precision in partial shape matching.
\end{abstract}

Key words: partial matching; feature fusion; convolutional neural networks; non-rigid 3D shape

收稿日期: 2020-06-22; 修回日期: 2020-09-10. 基金项目：国家自然科学基金(61702246); 辽宁省科技厅项目(2020JH4/10100045); 辽 宁省教育厅项目(603200052096); 大连市科技创新基金(2019J12GX038). 韩丽(1973-), 女, 博士, 教授, 硕士研究生导师, 主要研究方向为 计算机图形学、计算机视觉、人工智能; 倐宇宁(1995-), 女, 硕士研究生, 主要研究方向为计算机图形学; 朴京钰(1995一), 女, 硕士 研究生, 主要研究方向为计算机图形学; 徐圣斯(1992-), 男, 硕士研究生, 主要研究方向为计算机图形学; 王晓旻(1995一), 女, 硕 士研究生, 主要研究方向为计算机图形学; 兰鹏燕(1995-), 女, 硕士研究生, 主要研究方向为计算机图形学; 于冰(1988一), 男, 硕 士研究生, 主要研究方向为计算机图形学. 
近年来，深度学习理论在计算机视觉、自然语 言处理等领域得到了成功的应用, 如何将其有效 地推广至三维流形分析以及三维形状的匹配与检 索, 已经成为广大学者关注的重要问题. 在海量的 三维模型中, 非刚性三维模型因其变化多样, 更加 真实地描述现实世界，被广泛应用于各个领域. 然 而, 由于其具有变形、扭曲和延伸等复杂结构变换, 对模型的形状分析与匹配难度更大，更具有挑战性.

目前，广泛研究的非刚性三维模型匹配与检 索方法大多基于手工提取特征描述符，通过特征 之间的距离度量, 实现全局形状的相似性分析. 然 而，在现实生活中，不完整或局部相似性的模型普 遍存在，高效的三维形状局部匹配方法不仅是三 维模型检索技术的关键内容, 而且是实现形状编 辑、形状合成以及形状修复等技术的重要基础. 因 此，探索精准、高效的三维模型局部匹配方法具有 重要的科研意义.

本文深人结合深度学习理论，提出了一种基于 级联卷积神经网络 (convolutional neural networks, $\mathrm{CNN}$ )深度特征融合的三维形状局部匹配方法. 采 用特征袋模型理论，提出了几何图像构建方法，建 立稳定且具有区分力的特征矩阵; 进而，构建级联 $\mathrm{CNN}$ 框架，自动提取深度特征，并通过局部特征 与全局特征的深度学习实现点特征的融合及细化, 生成具有识别力的形状描述符; 最终, 提出了交叉 矩阵的相似性度量策略, 实现模型的局部结构识 别与匹配.一系列的实验验证了本文方法的高效 性与稳健性.

\section{1 相关工作}

三维模型匹配大致分为全局形状匹配与局部 形状匹配 2 个方面.

近年来，全局形状匹配研究已经取得了较为 成熟的研究成果. 在刚性模型的全局匹配研究中, Besl 等 ${ }^{[1]}$ 最先提出的迭代最近点 (iterative closest point，ICP)方法，通过反复迭代最小化采样点之间 的距离, 实现模型匹配. Rogers 等 ${ }^{[2]}$ 结合欧几里得 度量、形状上下文和图像相关特征，提出了一种距 离度量方法，提高了 ICP 的鲁棒性. Bronstein 等 ${ }^{[3]}$ 将目标模型通过刚性变换直接嵌人到源模型，不 仅解决了因为空间变换引入的误差问题，还将模 型的匹配问题转变成最优求解问题. 针对非刚性 三维模型的全局形状匹配，Osada 等 ${ }^{[4]}$ 提出了形状
分布描述符, 但是对于顶点较多的不相似模型, 也会产生相似的特征描述符. Ovsjanikov 等 ${ }^{[5]}$ 提出 了一个函数映射方法，通过函数空间的线性算子 有效地实现了等距变换形状的全局对应关系.

与全局形状匹配相比, 由于涉及更加复杂的 结构与不完整的局部性，三维模型的局部形状匹 配具有更大的难度和挑战性.

在局部形状匹配中，常见策略是将形状嵌人 到另一个域，通过搜索最近邻获得对应关系. Bronstein 等 ${ }^{[3]}$ 提出了直接嵌人源形状的广义多维 尺度(generalized multidimensional scaling, GMDS) 方法, 解决了部分形状匹配问题，但其中的局部 形状只是完整形状的一部分. Kim 等 ${ }^{[6]}$ 提出了利用 莫比乌斯变换和点投票方法实现等距形状匹配， 然而获得的是特征点之间的稀疏对应，而非形状 的对应; 而且，该方法具有较高的时间复杂度.

Sahillioğlu 等 ${ }^{[8]}$ 提出了尺度不变等距形状匹配 (isometric shape matching, ISM)方法, 利用投票策 略、根据肢体形状选择针点, 对部分形状和完整形 状进行均匀采样, 找到它们的对应关系. 但该方 法的性能在很大程度上取决于针点的选择, 并且 仅适用于与整个形状有一半或更多重叠的部分形 状匹配.

Barequet 等 ${ }^{[8]}$ 提出了利用模型拓扑结构的检索 方法, 但其拓扑结构提取一般对噪声敏感, 难以 获得稳定的部件分割结果. Shilane 等 ${ }^{[9-10]}$ 提出了优 先队列比较法, 首先在模型上生成大量采样点, 然后提取采样点周围的形状特征构建一个优先级 队列，并以选取形状区别力强的向量来计算相似 度. 但其特征提取和区分度排序的计算量较大, 难以应用于检索结构复杂的非刚性模型. Ferreira 等 ${ }^{[11]}$ 利用层次分割树将网格模型迭代地分为子部 件，再进行相似性匹配，但模型分割的效果会随 着模型的复杂度而改变. Tang 等 ${ }^{[12]}$ 利用三维模型 的稳定几何质心特征进行编码，以提取特征描述 符, 但是需要用从每个体素提取的几何质心和点 密度特征构造描述符, 时间复杂度较高.

最近，神经网络在图像图形分类和检索中的 成功应用引起了众多学者的关注, 很多学者将深 度学习理论引人三维形状分析中. $\mathrm{Su}$ 等 ${ }^{[13]}$ 从不同 角度对三维模型进行渲染, 将得到的二维渲染图 作为训练数据通过卷积网络进行训练, 训练出的 结果对三维模型的识别有比较好的效果. Wang 等 ${ }^{[14]}$ 提出基于八叉树 $\mathrm{CNN}$ 的三维形状分析，对体素化 
后的八叉树模型使用 $z$ 阶曲线( $(z-$ order curve)这种 方式进行存储，通过简单的计算快速求取索引的 值, 很好地契合了卷积的运算方式和规则. Qi 等 ${ }^{[15]}$ 提出一个层级的神经网络, 递归地应用在嵌套划 分的输人点云集，通过探索度量空间的距离，网 络结构可以随着上下文尺度的增加学习局部特征, 适应性地组合不同尺度的特征. 然而，面向局部 结构识别、大尺度非刚性变换和残损模型的深度学 习方法的研究仍然不够广泛.

本文充分利用图像的数据资源以及 $\mathrm{CNN}$ 学习 框架，提出了基于级联 $\mathrm{CNN}(\mathrm{F}-\mathrm{PointCNN})$ 深度特 征融合的非刚性三维模型局部匹配方法, 如图 1 所 示，重点解决大尺度非刚性变换和不完整模型的 局部形状匹配的关键问题.

首先，提取模型的低层几何特征：平均测地 线距离(average geodesic distance, AGD)特征与热 核(heat kernel signature, HKS)特征，构造互补的联 合形状描述; 其次, 利用特征袋 (bag of feature, $\mathrm{BoF})$ 模型, 构造几何图像的特征矩阵表示, 并通 过特征袋卷积神经网络(bag of feature-convolutional neural networks, BoF-CNN ${ }^{[16]}$ 优化训练, 提取非刚 性三维模型的深度全局特征; 进而，构建全局特 征与局部特征联合的点特征表示, 通过点卷积神 经网络(point-convolutional neural networks, Point$\mathrm{CNN}$ )细化，生成具有区分性的深度融合点特征. 本文方法提取的深度点特征不仅揭示了非刚性模 型的内蕴结构性, 而且对于大尺度残缺和拓扑变 换的局部模型具有极强的鲁棒性. 实验结果表明, 本文方法不仅能有效地识别大尺度非刚性变换的不 完整模型，而且有效地提高了模型局部匹配精度.

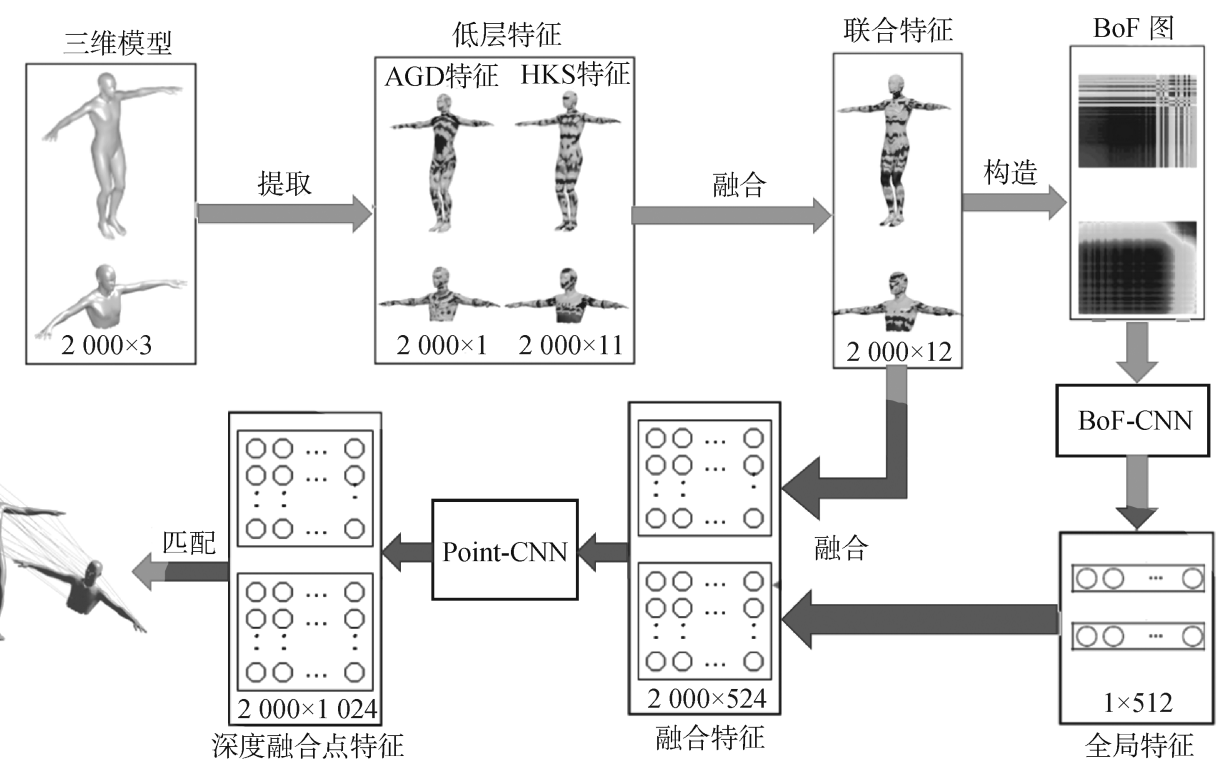

图 1 深度特征融合 F-PointCNN 的非刚性三维模型局部匹配方法

\section{2 几何特征学习的 BoF-CNN}

本文方法充分利用 CNN 在图像识别中的优 势，提出了基于几何图像的三维形状深度学习方 法. 利用低层几何特征构建互补的形状描述符, 生成统一的几何图像. 基于三维模型的几何图像 数据构建 $\mathrm{CNN}$ 学习框架, 实现深度全局特征的自 动提取与形状识别.

\section{1 几何图像表示}

特征袋模型(bag of feature, BoF)是词袋模型 (bag of words, BoW)的一种改进, 其将低层特征通 过聚类方法生成码本，然后将模型表示成一组码本
分布频率值的无序集合，有效地提高了特征的表示 能力，被广泛应用于图形图像及视频检索中 ${ }^{[17-21]}$.

本文方法充分利用 BoF 模型的思想，通过提 取非刚性三维模型的低层几何特征(AGD 和 HKS), 构建具有区分力的 BoF 几何图像, 有效地揭示大 尺度非刚性变换及局部模型的内蕴结构性.

之所以选择 AGD 和 HKS 特征, 主要是由于 AGD 可以有效地度量多分辨率模型的整体拓扑特 性，对于姿态变化不敏感 ${ }^{[21]}$; 而 HKS 依据时间尺 度可描述图形的多尺度局部特征, 对于局部扰动 具有鲁棒性 ${ }^{[22]}$.

因此，联合 AGD 与 HKS，构建非刚性模型的 
形状描述 $S^{\mathrm{HA}}\left(x_{i}\right)=\left(S_{\mathrm{HKS}}\left(x_{i}\right), S_{\mathrm{AGD}}\left(x_{i}\right)\right)$.

图 2a 所示为非刚性模型及其不同尺度残损结 构的 AGD 分布; 图 $2 \mathrm{~b}$ 所示为 $t=0.68$ 时非刚性变 换和残损模型上的 HKS 分布. 可见，无论是 AGD 还是 HKS 描述符，它们对非刚性变换及不同完整 程度模型具有良好的稳定性.
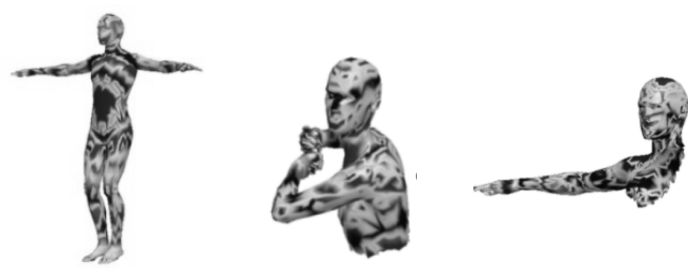

a. 不同姿态及局部非刚性模型的 AGD 分布
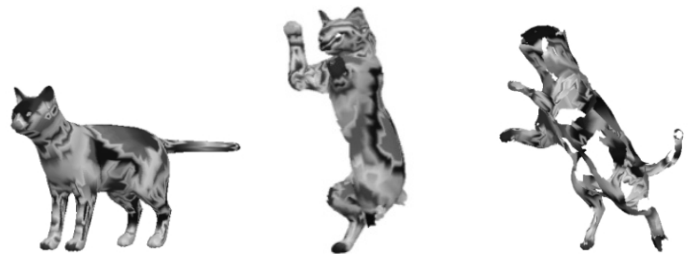

b. 不同姿态与局部和孔洞模型的 HKS 分布

图 2 不同姿态及不完整模型的 AGD 和 HKS 分布

本文采用 $\mathrm{BoF}$ 模型，利用 $k$-means 聚类方法对 特征 $S^{\mathrm{HA}}\left(s_{1}, s_{2}, \cdots, s_{n}\right)$ 进行聚类, 每个聚类中心定 义为视觉词汇, 则 $k$ 个聚类中心构建了模型的视觉 字典 $V=\left\{v_{1}, v_{2}, \cdots, v_{k}\right\}$; 进而, 模型中的每个特征 $s_{i}$ 量化到视觉词汇上进行特征编码, 构建 $k \times n$ 维特 征矩阵 $\boldsymbol{U}=\left\{u_{r 1}, u_{r 2}, \cdots, u_{r n}\right\}$. 其中,

$$
u_{r i}=\frac{\exp \left(-\alpha\left\|s_{i}-v_{r}\right\|_{2}^{2}\right)}{\sum_{l=1}^{k} \exp \left(-\alpha\left\|s_{i}-v_{l}\right\|_{2}^{2}\right)} .
$$

$k$ 为聚类中心数目; $n$ 为顶点数目; $\alpha$ 为平滑参数.

最终，通过视觉字典上的同一词汇进行特征 编码汇集, 将三维模型表示成一个无序局部特征 集的 BoF.

由于 $\mathrm{BoF}$ 仅表示了视觉词汇的频率信息，忽 略了词汇的空间关系，相关研究引人了测地线核 距离与双调和距离的思想 ${ }^{[20-21]}$ 构建形状上下文信 息, 以提高 BoF 的表示能力. 其中, 测地线核距离 对拓扑噪声敏感; 双调和距离对噪声和拓扑变化 具有鲁棒性，并且具有全局形状感知，提高了特 征的表达能力. 因此, 采用双调和距离构建全局 的 $\mathrm{BoF}$ 矩阵 $\boldsymbol{F}=\boldsymbol{U} \boldsymbol{K} \boldsymbol{U}^{\mathrm{T}}$. 其中, $\boldsymbol{K}$ 为双调和距离, $K_{i j}=\sum_{l=1}^{m} \frac{1}{\lambda_{l}^{2}}\left(\phi_{l}(i)-\phi_{l}(j)\right)^{2}$ 是由任意一对网格顶点 $i$ 和 $j$ 之间的 $m$ 个特征值 $\lambda$ 和特征向量 $\boldsymbol{\phi}$ 定义. 最终, 生 成 $(k \times k)$ 维全局的 $\mathrm{BoF}$ 矩阵 $\boldsymbol{F}$, 其不仅揭示了模型 的统一内蕴结构性，而且在不同拓扑与非刚性变 换模型中具有极强的稳定性.

图 3 所示为不同姿态残缺模型的 BoF 矩阵及 分布曲线. 可见，在不同姿态模型中，无论是 $\mathrm{BoF}$ 矩阵还是曲线都有效地揭示了内蕴结构的一致性; 同时, 在具有孔洞拓扑变化的模型中也体现了极 强的稳定性. BoF 矩阵不仅揭示了同类异构模型的 一致性，也突显了不同种类模型的差异性，更可 以对不同分辨率的非刚性模型生成统一的 BoF 表 示 $(k \times k), k$ 为聚类中心数目, 也为特征维度.

\section{2 基于几何图像 CNN 学习模型}

为了有效地获取海量三维模型的高层语义结 构特征，本文进一步基于全局 BoF，建立学习框架 BoF-CNN ${ }^{[1]}$, 自动提取深度特征, 提高特征的识别 与表示能力.

如图 4 所示, 本文的 BoF-CNN 采用了 VGG-M 卷积网络架构思想, 包括 5 个卷积层, 2 个全连接 层以及 1 个 Softmax 输出层. 所有卷积操作中的卷 积核大小为 $3 \times 3$, 步长为 1 , 每层采用 $R e L U$ 激活函 数及最大池化操作. 选择 ReLU 激活函数是因为它 可以使网络训练更快，在增加非线性可分性的同 时, 可以防止梯度消失. 最终, 采用交叉损失函数 进行学习训练与优化.

具体方法可归纳如下:

方法 1. 基于几何图像 CNN 的形状分类.

输人. 三维非刚性模型 $D=\left\{M_{1}, \cdots, M_{m}\right\}$.

输出. 512 维深度特征, 分类结果.

For $i=1: m$

计算模型 $M_{i}$ 的 $1 \times n$ 维的 $\mathrm{AGD}$ 特征 $s_{i}^{\mathrm{a}}$;

计算模型 $M_{i}$ 的 $q \times n$ 维的 HKS 特征 $s_{i}^{\mathrm{h}}$;

构造 $(q+1) \times n$ 维的多特征表示 $s_{i}^{\mathrm{HA}}$;

通过 $k$-means 建立 $(q+1) \times k$ 词典和 $k \times n$ 维 $\boldsymbol{U}_{i}$;

通过双调和距离生成 $k \times k$ 维的 BoF 矩阵 $\boldsymbol{F}_{i}$; end for

将 $\mathrm{BoF}$ 矩阵 $\boldsymbol{F}_{i}$ 转换为 $\mathrm{BoF}$ 图像 $B_{i}(i=1,2, \cdots, m)$;

While ( $i \leqslant T)$ do (其中, $T$ 为采样点个数)

输人特征图像 $B_{i}(i=1,2, \cdots, m)$;

BoF-CNN 中学习并提取 512 维特征向量 $\boldsymbol{V}\left(B_{i}\right)$; 在 Softmax 层对 $B_{i}$ 进行分类预测，得到 $\hat{p}\left(B_{i}\right)$; 计算分类精度 $A(i)$; 交叉熵损失函数优化; $i++$

输出分类精度 $A(i)$ 以及深度特征 $\boldsymbol{V}\left(B_{i}\right)$. 


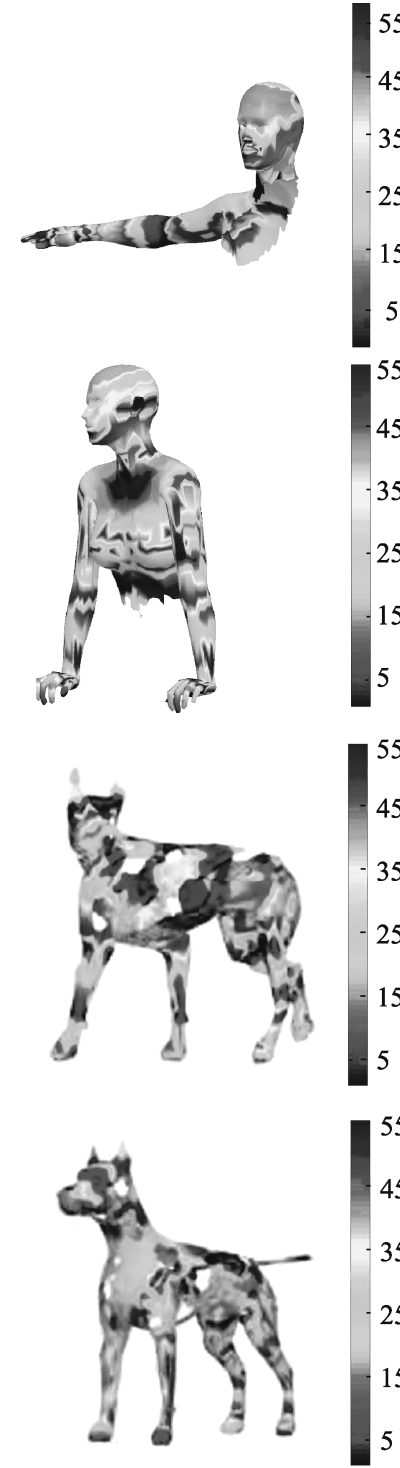

a. 残缺模型
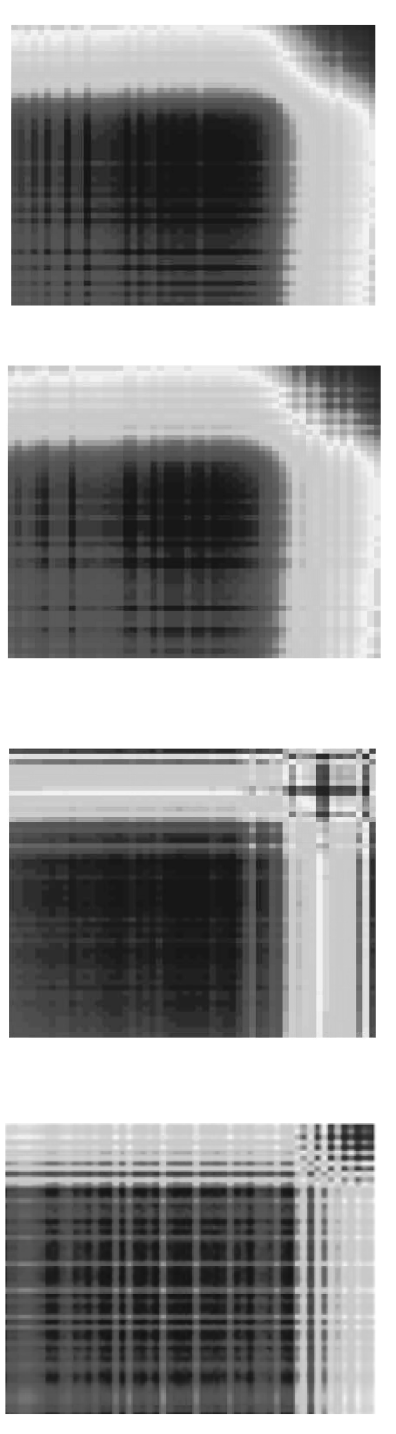

b. 特征矩阵
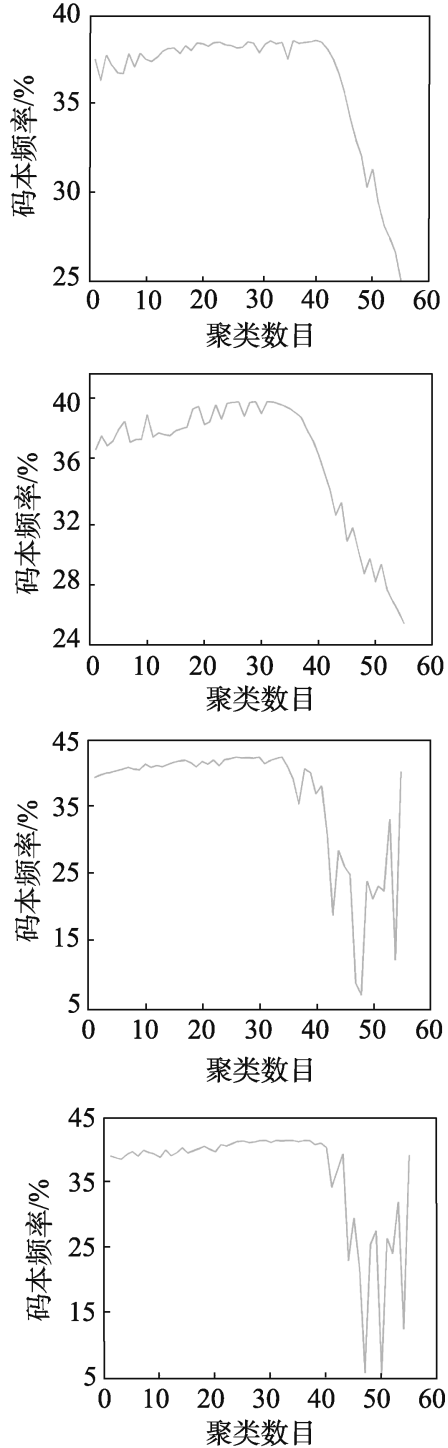

c. 分布曲线

图 3 不同姿态残缺模型的 $\mathrm{BoF}$ 特征矩阵及分布曲线

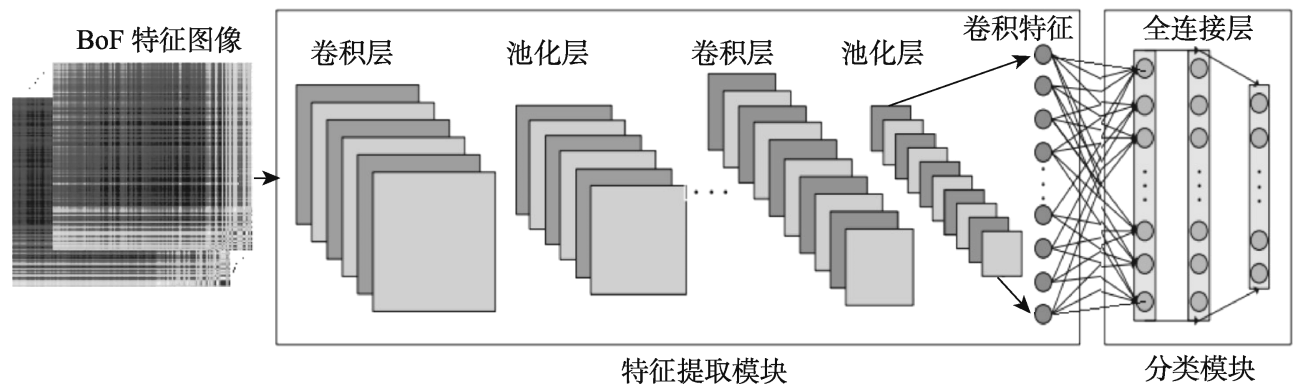

图 4 BoF-CNN 深度学习框架

实验中，首先采用 ImageNet 中充分的图像资 源进行预训练, 以三维模型数据库 SHREC2010, SHREC2011, SHREC2015, SHREC2016生成的 BoF 图像 $(224 \times 224$ 像素 $)$ 作为输人进行微调.

随机选取 $70 \%$ 的数据集作为训练样本, 剩下 $30 \%$ 的数据集作为测试样本. 为了减少随机选择
造成的误差, 采用 1000 次的模型迭代训练, 并取 平均值作为最终的数据集分类精度.

图 5 所示为在 SHREC2011 数据库上的分类结 果. 可见, 在迭代次数达到 800 次时, 网络训练准 确率和交叉损失率基本收敛.

表 1 所示为不同三维模型数据库的形状分类 


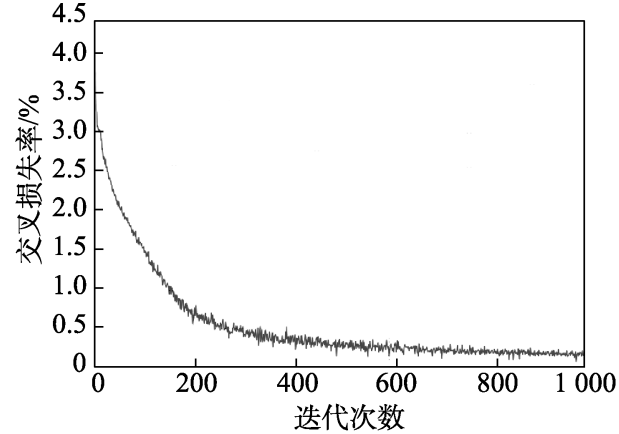

a. 交叉损失率

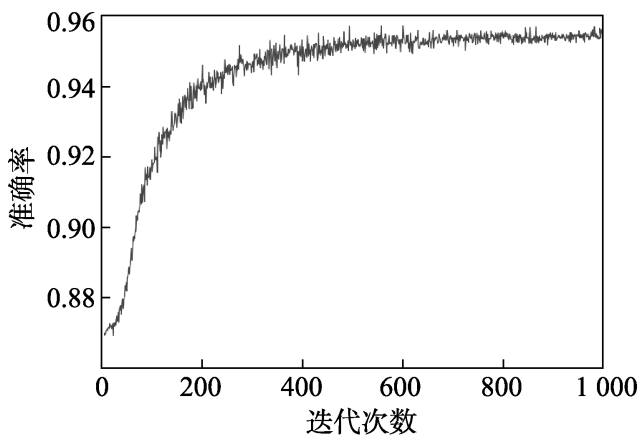

b. 分类训练准确率

图 5 SHREC2011 数据集上 BoF-CNN 分类结果

表 1 在不同模型数据库上的分类精度

\begin{tabular}{ccc}
\hline 模型数据库 & BoF-CNN & F-PointCNN \\
\hline SHREC2010 & 93.75 & 97.16 \\
SHREC2011 & 95.50 & 98.43 \\
SHREC2015 & 95.47 & 96.25 \\
SHREC2016 & 86.11 & 91.20 \\
\hline
\end{tabular}

精度. 可见，基于 BoF-CNN 提取的深度特征具有 显著的区分性，能够有效地识别结构复杂以及拓 扑变化的非刚性三维模型, 在三维数据库 SHREC2010, SHREC2011 与 SHREC2015 中体现出 较好的识别性能. 然而, 在结构残损与具有孔洞的 模型数据库(SHREC2016) 中存在一定的局限性, 主要由于 BoF-CNN 学习的是全局深度特征, 不完 整模型丢失了大部分结构信息，同时具有大尺度 的孔洞, 因此无法实现有效的局部结构识别.

为了弥补几何图像特征在识别大尺度残损模 型及局部结构的局限性，本文进一步提出一种基 于深度点特征融合学习机制, 有效地提高大尺度 非刚性变换局部形状的识别与匹配性能.

\section{3 深度特征融合的非刚性模型匹配方法}

本文基于 PointNet ${ }^{[16]}$ 学习模型的思想, 构建 深度融合点特征学习模型 F-PointCNN, 通过交叉 矩阵度量, 高效实现局部结构的识别与匹配.

\section{1 深度融合点特征学习模型 F-PointCNN}

首先, 输出 BoF-CNN 学习框架中的全局深度 特征，与模型中顶点的局部特征 AGD 和 HKS 相关 联，构建点的融合特征表示 $(n \times 524)$; 进而, 将点 特征输人到 Point-CNN 中细化提纯; 最终, 获得信 息丰富的深度融合点特征 $n \times 1024$, 如图 6 所示.

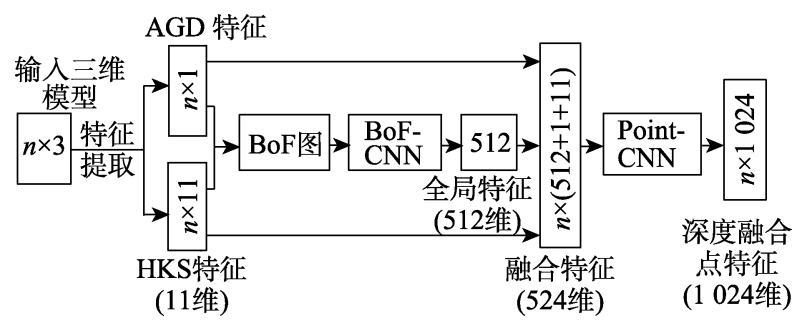

图 6 F-PointCNN

由于三维模型具有复杂结构以及分布不规则 特性, 为了获取多尺度点特征表示, Point-CNN 采 用 AlexNet 框架思想, 基于尺度不同的卷积核提取 点的深度特征. 该网络由 5 个卷积层和 3 个全连接 层构成学习网络, 分别设置卷积核为 $(11 \times 11)$, $(5 \times 5),(3 \times 3)$. 其中, 第 6 层与第 7 层为全连接层, 第 8 层为 Softmax 层输出分类结果.

本文以 BoF-CNN 的输出特征与 $n$ 个顶点的局 部特征关联, 构造 $n \times 524$ 的特征矩阵作为输人, 通过随机梯度下降函数训练及优化, 获得最优的 分类输出结果. 最终, 将训练好的网络学习模型的 第 7 层特征作为深度融合的点特征输出, 用于实现 模型的匹配与检索.

基于 F-PointCNN 学习框架输出的点特征, 融 合了模型的局部特征与全局特征, 大大提高了非 刚性变换模型以及局部残损模型的识别精度. 如 表 1 所示, 对于完整模型数据库(SHREC2010, SHREC2011，SHREC2015)分类精度平均提高了 $2.37 \%$, 而对于不完整模型数据库 SHREC2016 提 高了 $5.09 \%$.

\section{2 相似性匹配}

基于深度融合的点特征表示，本文进一步提 出交叉矩阵检测方法, 有效地实现局部模型的形 状匹配.

首先，采用最远点采样(farthest point sampling, FPS $)^{[23]}$ 在模型上选取 $T$ 个针点, 如图 7 所示. 计算 每个顶点到针点的距离, 建立 $T$ 维嵌人特征表示, 有效地将高维深度融合点特征转换到低维嵌人空间

$$
\left\{\begin{array}{l}
D_{X}\left(x_{i}\right)=\sum_{k=1}^{T} \frac{1}{\omega} d\left(x_{i}-\hat{x}_{k}\right) \\
D_{Y}\left(y_{j}\right)=\sum_{k=1}^{T} \frac{1}{\omega} d\left(y_{j}-\hat{y}_{k}\right)
\end{array} .\right.
$$


其中, $x \in \mathbb{R}^{n \times p}, y \in \mathbb{R}^{m \times p}$ 为 F-PointCNN 输出模型 $X$ 与 $Y$ 的深度融合特征; $m$ 与 $n$ 分别为模型顶点的 数目; $p=1024$ 为点的特征维度. $D_{X}\left(x_{i}\right)$ 为模型 $X$ 的顶点在 $T$ 维 $(T \ll 1024)$ 嵌人空间的度量, 即模型 上任意顶点 $x_{i}$ 到 $T$ 个针点 $\hat{x}_{k}$ 的加权测地距离 $d\left(x_{i}, \hat{x}_{k}\right)$, 所有点的嵌人度量为 $D(x)$. 权值 $\omega$ 根 据顶点 $x_{i}$ 和针点 $\hat{x}_{k}$ 的余弦距离计算. 距离越小, 模型越相似，权值越大；反之，权值越小.

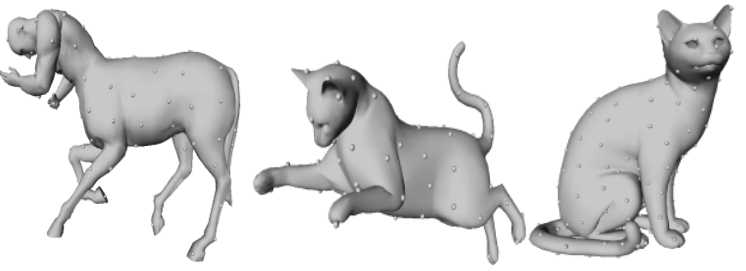

图 7 不同模型的针点选取, $T=100$

设 $\left(x_{i}, x_{j}\right)$ 与 $\left(y_{k}, y_{t}\right)$ 为模型 $X$ 与 $Y$ 中对应点, 则 模型间的相似距离 $d_{\text {sim }}(X, Y)$ 满足

$$
d_{\text {sim }}(X, Y)=\min \left|D_{X}\left(x_{i}-x_{j}\right)-D_{Y}\left(y_{k}-y_{t}\right)\right| .
$$

其中, $D_{X}\left(x_{i}-x_{j}\right)$ 为模型 $X$ 中 2 点 $x_{i}$ 和 $x_{j}$ 在嵌人 空间的欧几里得度量; 同理, $D_{Y}\left(y_{k}-y_{t}\right)$ 为模型 $Y$ 中 2 点 $y_{k}$ 和 $y_{t}$ 在嵌人空间的欧几里得度量.

为了有效地计算模型的最小相似距离，本文 提出了交叉矩阵检测方法，通过计算 2 个模型中的 互距离 $\boldsymbol{D}_{\mathrm{mut}}(X, Y)$ 和 $\boldsymbol{D}_{\mathrm{mut}}(Y, X)$, 建立模型相似 性度量矩阵 $\boldsymbol{D}_{\mathrm{Sim}}(X, Y) \in \mathbb{R}^{n \times m}$ ，即

$$
\left\{\begin{array}{l}
\boldsymbol{D}_{\text {mut }}(X, Y)=\sum_{i=1}^{n} \sum_{k=1}^{T} \frac{1}{\omega} d\left(x_{i}-\hat{y}_{k}\right) \\
\boldsymbol{D}_{\text {mut }}(Y, X)=\sum_{i=1}^{m} \sum_{k=1}^{T} \frac{1}{\omega} d\left(y_{i}-\hat{x}_{k}\right) \\
\left.\boldsymbol{D}_{\text {Sim }}(X, Y)=\frac{\boldsymbol{D}(X) \boldsymbol{D}(Y)^{\mathrm{T}}}{\boldsymbol{D}_{\text {mut }}(X, Y) \boldsymbol{D}_{\text {mut }}(Y, X)^{\mathrm{T}}}\right)
\end{array} .\right.
$$

通过搜索相似性度量矩阵 $\boldsymbol{D}_{\mathrm{Sim}}(X, Y)$ 中每行 的最小值，获得模型间的最佳匹配点对.

图 8 所示为基于交叉距离矩阵检测所获得的 不同姿态的猫模型的局部点匹配结果.

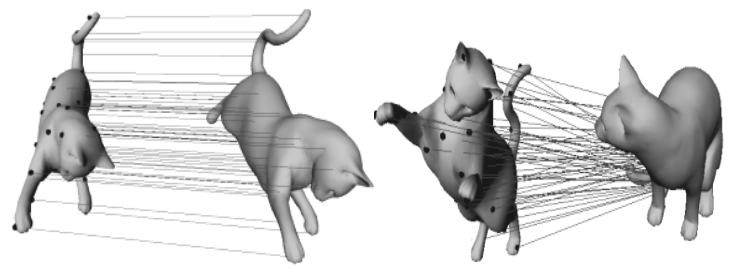

图 8 不同姿态非刚性模型的匹配点对

\section{4 实验结果分析}

本文实验平台为 $3.2 \mathrm{GHz}$ Pentium CPU, $8 \mathrm{~GB}$ 内存的 $\mathrm{PC}$, 实验数据集来自公开的非刚性模型 数据库 SHREC2010, SHREC2011, SHREC2015 和 SHREC2016. 其中, SHREC2010 包含 10 类共 200 个模型; SHREC2011 包含 30 类共 600 个模型; SHREC2015 包含 50 类共 1200 个三维模型; SHREC2016 分为孔洞和不完整 2 种不同的三维数 据, 包含 8 类共 400 个模型.

实验采用 ImageNet 数据库 1000 类数据进行 网络预训练, 然后使用三维数据进行微调.

首先，提取每个非刚性模型的 BoF 特征图像 $(224 \times 224)$. 在 BoF 图像生成中主要涉及拉普拉斯 特征求解、双调和距离计算以及视觉词汇量化, 因 此，其时间度复杂度为 $O\left(k n^{2}\right), k$ 为聚类中心数目; $n$ 为模型个数. 其次, F-PointCNN 学习与深度融合 特征提取中, 使用随机梯度下降函数优化学习网 络, 其中, 学习率设为 0.03 , dropout 为 0.5 , 正则化 权重为 $5 \times 10^{-4}$, 所有模型都采用 1000 次迭代的训 练, 通过训练学习与优化, 输出深度点特征; 最终, 基于交叉矩阵检测, 实现非刚性三维模型的局部 形状匹配.

模型形状匹配的过程中，利用最远点采样进 行特征降维, 并通过交叉矩阵度量实现最优匹配 点对的获取, 其时间复杂度为 $O(T m n)$, 其中, $T$ 为 采样点个数, $m$ 和 $n$ 分别为匹配模型的点数.

表 2 所示为本文方法在不同模型上的运行时 间. 其中, 用时最短的为 human 模型, 从几何图像 生成到特征提取与模型匹配约半分钟的时间; 时间 最长的是 David 模型, 总共为 $106.33 \mathrm{~s}$. 总体而言, 多数模型都能在 $1 \mathrm{~min}$ 之内完成特征提取与匹配.

表 2 不同模型的运行时间

\begin{tabular}{lrcc}
\hline \multirow{2}{*}{ 模型 } & \multirow{2}{*}{ 面片个数 } & \multicolumn{3}{c}{ 时间 $/ \mathrm{s}$} \\
\cline { 3 - 4 } & & $\mathrm{BoF}$ 图像生成 & 深度特征提取与匹配 \\
\hline cat & 7969 & 29.34 & $5.06+4.34$ \\
centaur & 7996 & 29.83 & $5.32+5.13$ \\
David & 100954 & 62.75 & $5.26+38.32$ \\
human & 3206 & 25.69 & $5.15+3.65$ \\
Victoria & 10000 & 30.13 & $5.13+10.78$ \\
\hline
\end{tabular}

采用普林斯顿基准协议，通过计算测地误差 度量模型的匹配质量.

设 $(x, y) \in X \times Y$ 为算法所获得的模型匹配点对, 而模型的真实匹配 (ground truth, GT) 关系为 
$\left(x, y^{*}\right)$. 其测地误差定义为

$$
\rho(X)=d_{N}\left(y, y^{*}\right) / S_{\text {area }}(X)^{\frac{1}{2}} .
$$

其中, $d_{N}$ 为 $y$ 和 $y^{*}$ 之间的测地距离; 而 $S_{\text {area }}(X)$ 为 模型 $X$ 的表面积. 测地误差 $\rho(X)$ 通过归一化距离 误差实现, 理想的测地误差等于零. 每一类的平均 测地误差是每一类模型的测地误差的平均值.

模型分类准确率为

$$
A(i)=\frac{\left|x: x \in X_{i} \wedge \hat{p}(x)=p(x)\right|}{\left|x: x \in X_{i}\right|} .
$$

其中, $\hat{p}(x)$ 和 $p(x)$ 分别为模型的实际标签和分类 方法的预测标签，预测标签与实际标签相同即为 分类正确.

分别基于 BoF 几何图像、BoF-CNN, F-PointCNN 学习框架进行形状识别的实验测试, 并围绕非刚 性模型的局部匹配，与相关研究进行了实验论证 与对比分析.

\section{$4.1 \mathrm{BoF}$ 几何图像}

基于模型的低层几何特征 AGD 与 HKS，构建 $\mathrm{BoF}$ 特征矩阵. 在 $\mathrm{BoF}$ 生成过程中, 采用 $k$-means 进行特征聚类, 将所有点与聚类中心进行特征量 化, 通过双调和距离构建了 $k \times k$ 维的 BoF 矩阵. 聚类中心数目 $k$ 的选择不仅影响词汇空间大小，还 决定 $\mathrm{BoF}$ 的维度. 因此, 采用不同聚类中心数目 $k=50,80,100,120,150$ 进行实验测试.

如图 9 所示, 纵坐标为识别误差, 横坐标为 训练及测试样本的比例. 分别以 SHREC2010 和
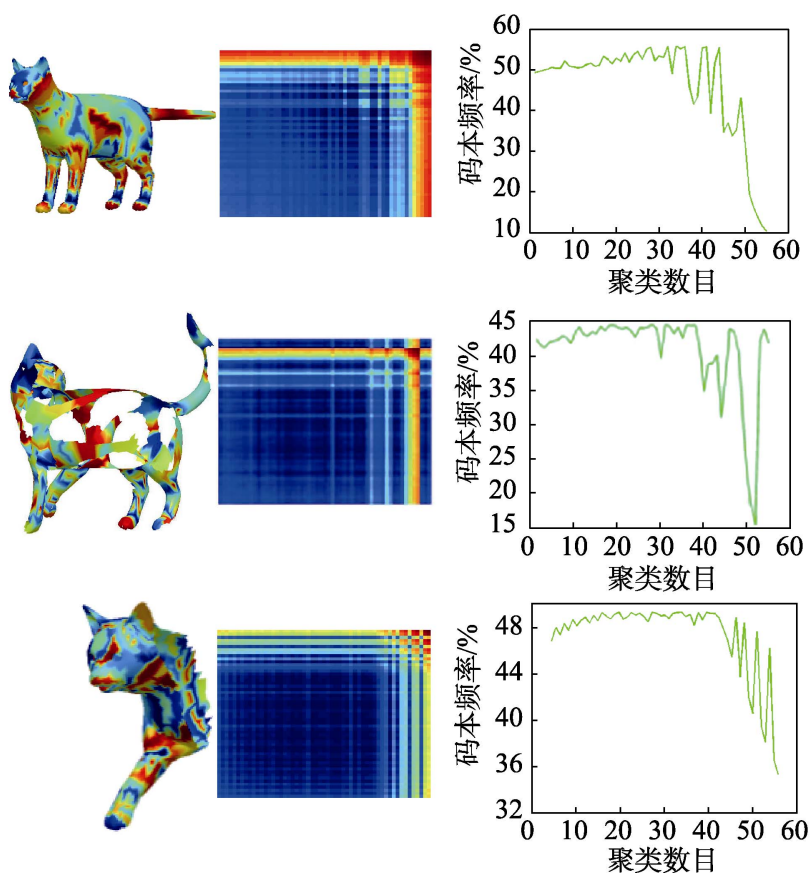

图 10 不同姿态、孔洞及残缺模型的 $\mathrm{BoF}$ 矩阵以及分布曲线
SHREC2011 模型数据库为例进行实验分析, 随着 训练集比例增大, 识别误差逐渐降低, 并在比例 大约为 0.7 时达到最低, 且 $k=120$ 的识别误差最小. 因此，在后续实验中，采用 $k=120$ 的聚类空间， $7: 3$ 的比例划分训练集与测试集.

图 10 所示为不同非刚性残缺模型的 BoF 矩阵 及其特征分布曲线. 可见，全局 BoF 不仅揭示了非
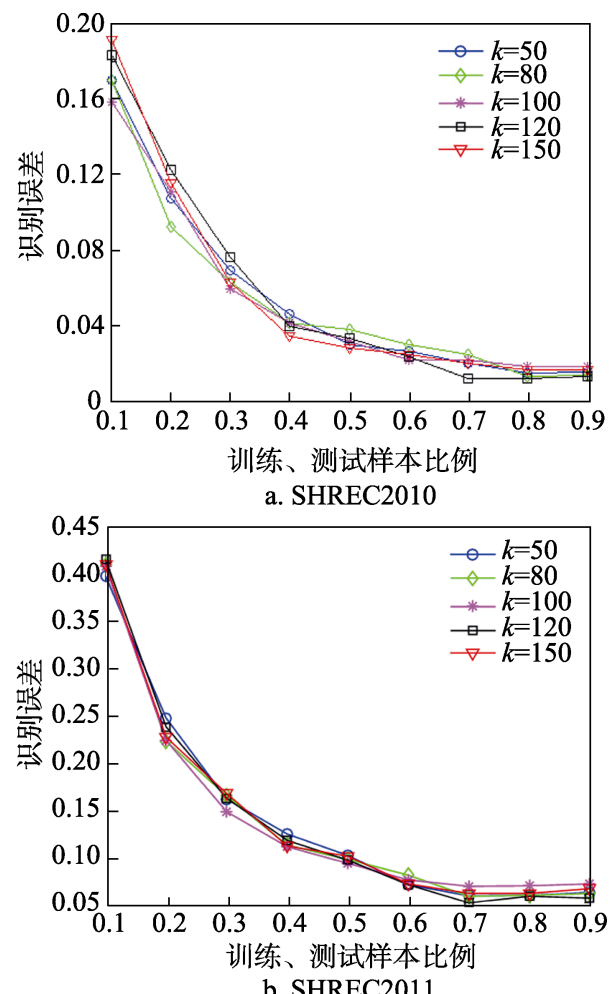

图 9 不同数据库上选择 BoF 维度 $k$ 下的识别误差
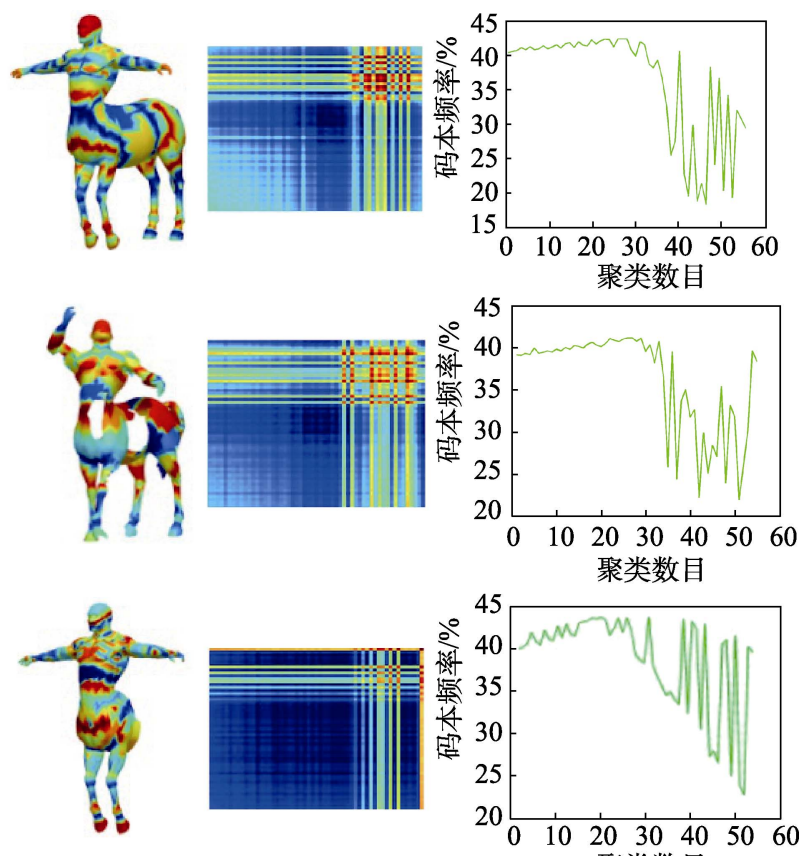

聚类数目 
刚性模型的内蕴结构一致性, 而且对于大尺度拓 扑变化孔洞及较小结构的局部模型体现出极强的 稳定性.

\section{2 基于 F-PointCNN 的特征学习框架性能}

本文的 F-PointCNN 学习框架采用了级联 $\mathrm{CNN}$ 结构，由 2 部分组成: BoF-CNN 用来实现全局 深度特征的提取, Point-CNN 则提取深度融合的点 特征.

实验采用公开的非刚性三维模型数据库 SHREC2015 和 SHREC2016，对比分析了基于 BoF 几何图像 ${ }^{[24]}$, BoF-CNN 的全局深度特征以及基于 F-PointCNN 融合特征的形状分类结果, 实验结果 如表 3 所示. 可见, 经过双重 CNN 学习的点特征 在识别精度上明显优于前 2 组数据.

图 11 所示为 F-PointCNN 在 SHREC2015(完整
模型)以及 SHREC2016(残损模型)上各个类的分类 结果. 可见, F-PointCNN 不仅能够有效识别大尺度 非刚性变换的模型，而且对于具有明显的拓扑结构 变化(hole)和大尺度残缺(cut)的数据也体现出良好 的识别性能. 图 11 中对角线为测试数据的分类精 度，黄色代表较高的精度，蓝色代表较低的分类精 度. 可见从整个大类中, 只有 $\operatorname{dog}$, wolf, cat 模型出现 了略微的混淆, 其他模型都具有较高的分类精度.

\section{表 3 不同框架的形状分类精度}

\begin{tabular}{lccc}
\hline \multicolumn{1}{c}{ 框架 } & SHREC2015 & SHREC2016(hole) & SHREC2016(cut) \\
\hline BoF $^{[19]}$ & 85.8 & 82.4 & 81.6 \\
BoF-CNN $^{[16]}$ & 95.5 & 90.7 & 89.4 \\
F-PointCNN & 96.2 & 92.1 & 90.3 \\
\hline
\end{tabular}

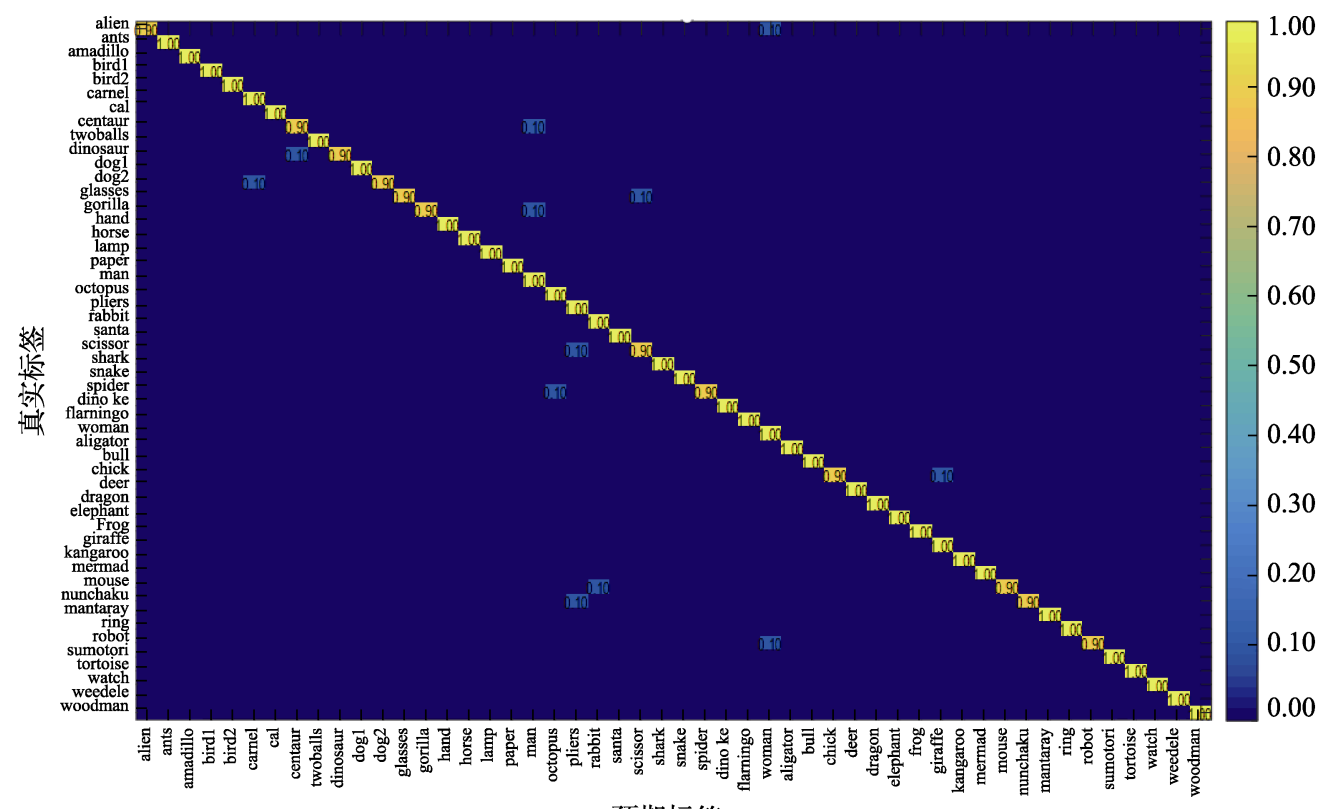

预期标签

a. SHREC2015 中各类模型

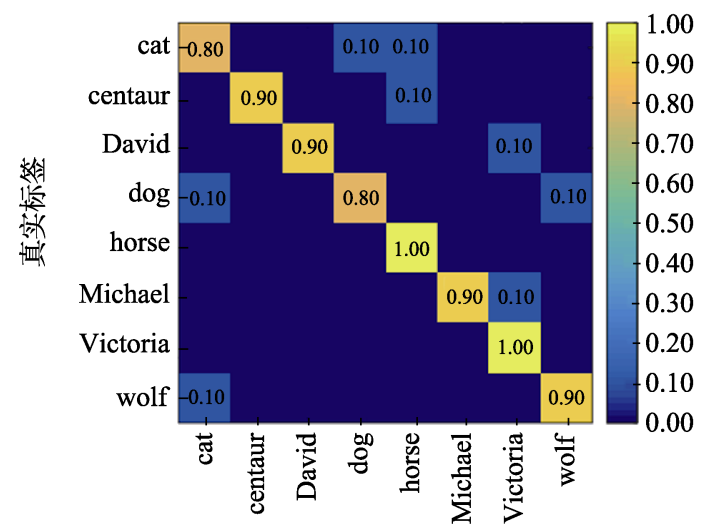

预期标签

b. SHREC2016 残损模型

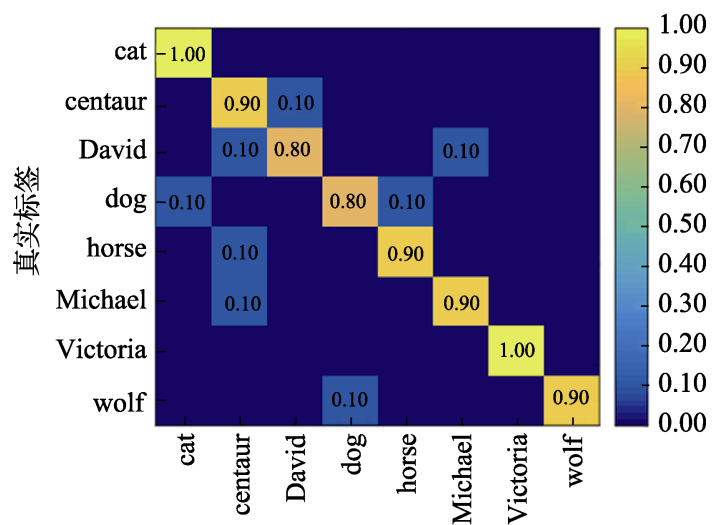

预期标签

c. SHREC2016 空洞模型

图 11 F-PointCNN 在不同数据库中各类的分类精度 


\section{3 局部形状匹配}

为了提高三维模型的匹配效率, 本文提出了 基于针点的交叉距离检测方法，利用模型的针点 建立点特征的多尺度度量空间，实现高效的形状 匹配.

采用最远采样点采样 FPS 获取均匀分布的针 点, 其在非刚性变换与噪声模型中具有很强的稳 定性，如图 12a 和图 12b 所示; 针点数逐渐增加 时，模型匹配的测地误差趋于稳定，如图 12c 所 示. 因此, 实验中设置 $T=200$, 建立模型的统一度 量空间.

在模型匹配中，分别进行全局匹配以及局部 匹配测试, 并将本文匹配方法与 ISM 方法 ${ }^{[25]}$ 、投 票方法 ${ }^{[26]}$ 进行对比分析. 如图 13 所示, 以 SHREC2016 模型数据库为例, 对孔洞残缺以及局 部结构残损模型进行了验证. 图 13a 所示为残损模 型与非刚性完整变换模型的局部匹配结果; 图 13b 所示为 2 组孔洞残损模型的匹配效果; 图 $13 \mathrm{c}$ 显示 了在 David, centaur 以及 cat 等姿态变换模型中的 局部匹配效果. 可见, F-PointCNN 具有显著的区分 力, 在残损及拓扑变化模型中具有极强的鲁棒性, 可有效地实现局部与完整、局部与局部、大尺度残 损模型之间的形状匹配.

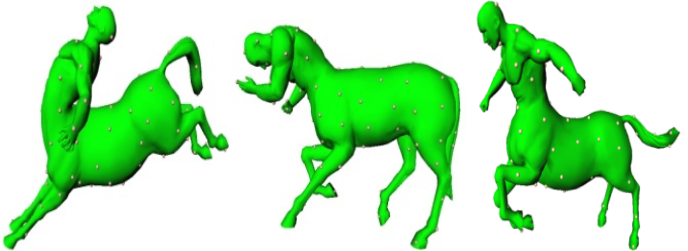

a. 不同姿态 centaur 模型的针点选取

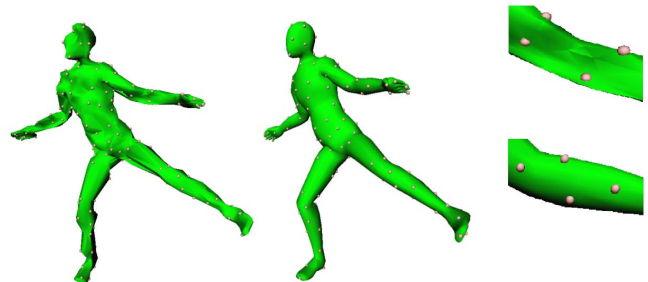

b. 不同高斯噪声 human 模型的针点选取

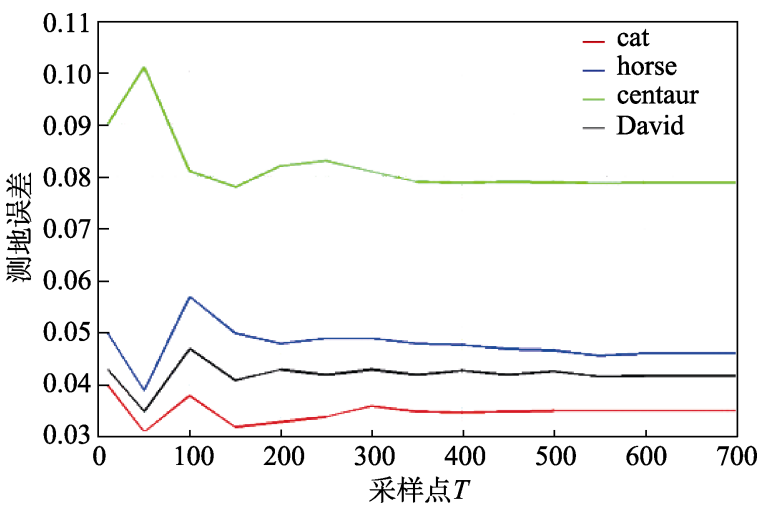

c. 测地误差

图 12 针点的选取及测地误差曲线

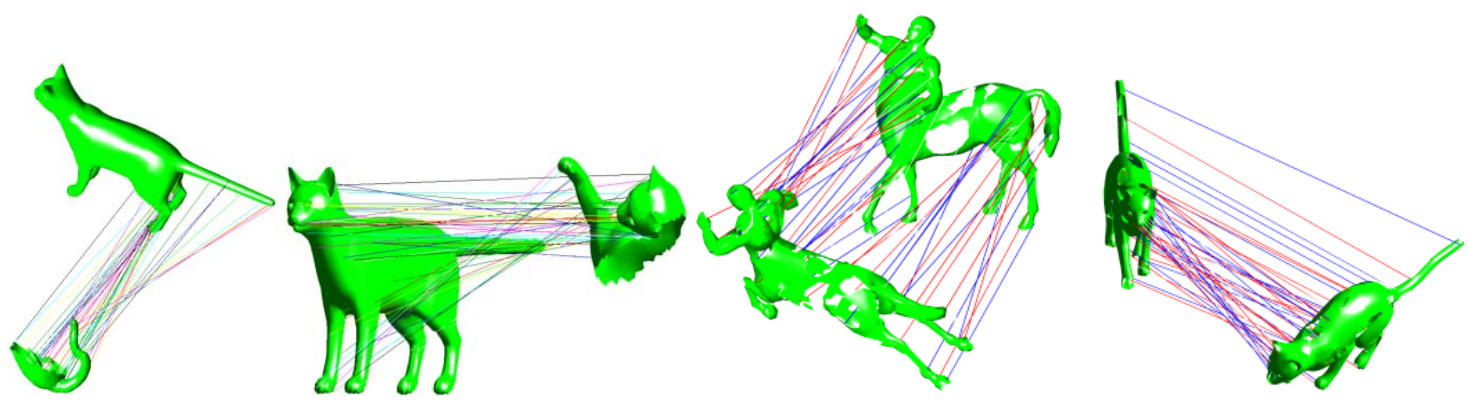

a. 残损部分与非刚性变换完整模型的局部匹配

b. 具有孔洞的非刚性模型全局匹配
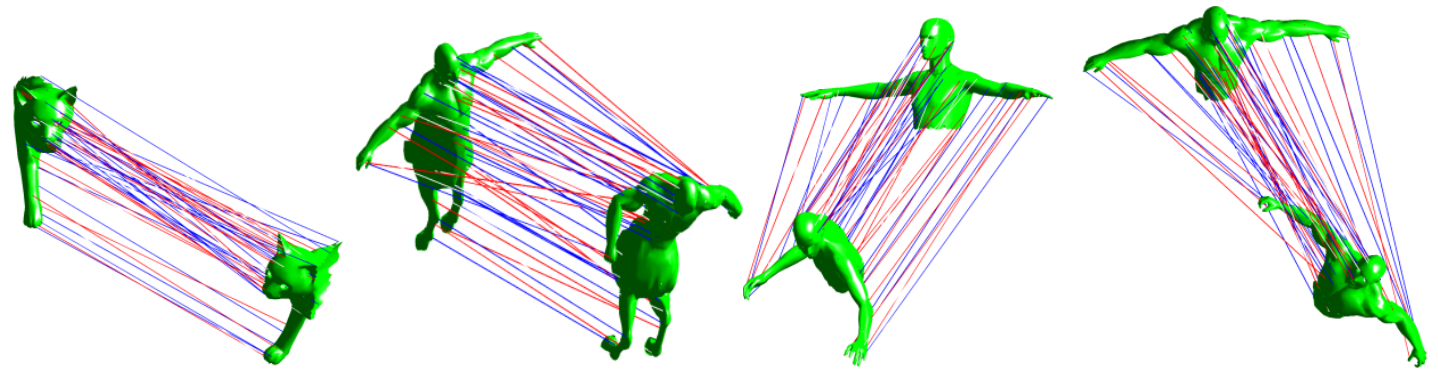

c. 残损非刚性模型的匹配

图 13 不完整非刚性模型的局部匹配 
最后，进一步将 F-PointCNN 与经典的 ISM 方 法和投票方法进行对比分析，如图 14 所示.
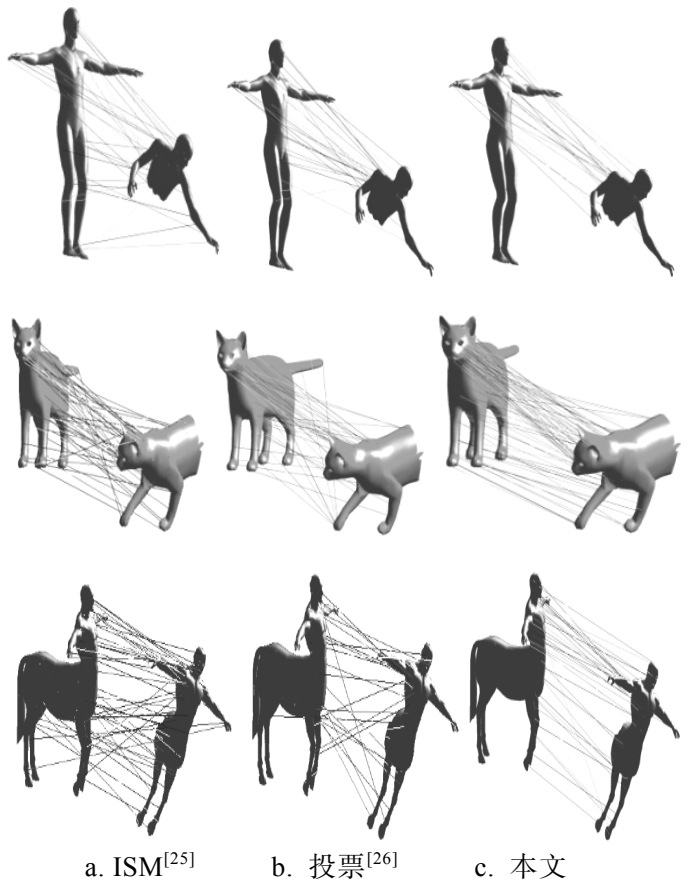

图 14 不同方法模型匹配比较

ISM 方法从网格模型中提取形状极值点作为 针点后，通过组合搜索最小化等距失真函数实现 粗匹配，进而通过密集采样按周围均匀密度分布 获取对应点将稀疏对应到密集匹配，从而实现模 型的局部匹配. 投票方法通过对模型之间的局部 对应进行粗采样后，采用等级-投票-组合的方法进 行正确的配准; 等级高的采样匹配具有更高的置 信度值，并累积到投票矩阵中，最后将累积的投票 组合形成采样点之间的对应，完成模型匹配. 然 而, 投票与 ISM 方法的匹配准确性在很大程度上 取决于采样点的选择，在局部形状与整体形状有 较大重叠时可取得较好的效果, 但在重叠较小(面 积小于 $50 \%$ )时，完整模型和残损模型上经常会找 不到一致的形状极值点，导致投票错误，进而影响 匹配的效果.

本文方法基于 $\mathrm{CNN}$ 框架自动提取点的深度融 合特征，并将点特征嵌人统一的空间进行度量与 交叉相似矩阵检测，从而获得最优的匹配点对。点 特征的获得不依赖于个别模型的局部采样，而是 数据驱动的自动学习，其特征表示具有极强的区 分性. 相似性度量亦在统一的尺度空间，具有极强 的稳定性. 如图 14 所示，在一致的数据搜索空间 与误差约束下，本文方法获得更为精准的模型匹 配结果.
图 15 所示为 5 种方法的累积曲线, 显示了测 地线误差与匹配的百分比. 其中, GD-DNA 方法 ${ }^{[27]}$ 即测地圆盘匹配方法, 解决了 ISM $^{[25]}$ 和投票 ${ }^{[26]}$ 方 法在大尺度局部残损模型匹配的局限性. 然而，其 只适用于大的封闭测地圆盘，小的测地圆盘或边 界都会影响匹配效果，因此无法有效地实现孔洞 模型的匹配. Non-rigid Puzzle ${ }^{[28]}$ 利用函数映射框 架，正则化求解预先已知的全模板模型之间的部 分函数对应关系，其局限性是必须在模型和查询 形状之间提供相应的函数.

本文方法从全局与局部特征出发, 利用级联 $\mathrm{CNN}$ 自动提取深度特征, 具有显著的区分性与稳 定性，无论是在大尺度变换模型还是较小残损模 型及孔洞模型，都具有良好的识别与匹配效果.

由图 15 可见，随着测地误差的提高，本文方 法与 Non-rigid Puzzle, GD-DNA 相似, 在较小误差 情况下 $(<0.13)$ 匹配精度逐步稳定. 而 ISM、投票方 法在测地误差为 0.20 以后趋于稳定. 同样, 无论是 在测地误差较小 $(<0.13)$ 还是相同的情况下，本文 方法在匹配精度上均具有更显著的优势.

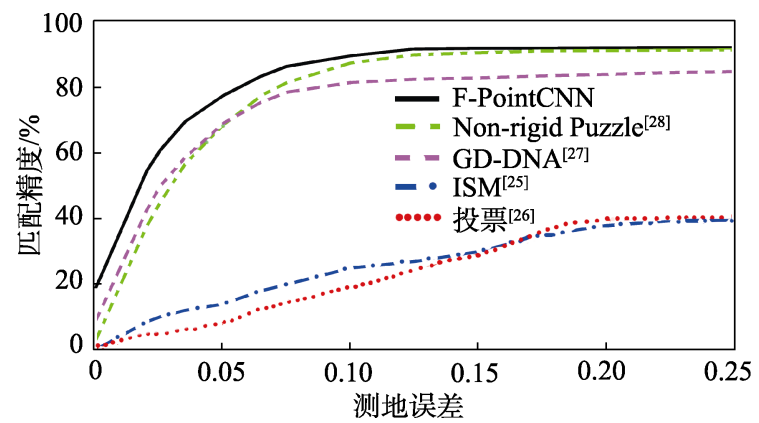

图 15 不同方法的测地误差及匹配精度

\section{5 结 语}

形状匹配是计算机图形学和视觉领域的一个 基本问题，在动画、纹理映射、机器人视觉和医学 成像等领域有着广泛的应用. 面向海量、复杂三维 模型的局部形状匹配需求，本文提出了基于级联 $\mathrm{CNN}$ 深度融合特征的非刚性三维形状局部匹配方 法，重点解决大尺度残损和孔洞模型的匹配问题.

本文首先提出了 $\mathrm{BoF}$ 几何图像生成方法, 有效 地揭示了不同拓扑变化及局部残损模型的内在结 构一致性. 进而，建立了 BoF-CNN 与 Point-CNN 联合学习机制，生成具有丰富信息的点深度特征 表示，有效地提高了特征的区分性，实验论证了其 在非刚性局部形状识别与匹配中的显著性能. 
然而，在低层几何特征提取与 $\mathrm{BoF}$ 构建中，需 要计算模型的邻接矩阵并进行特征分解, 增加了 时间复杂度. 尤其对于具有多个结构的不连通模 型，存在一定的局限性.

因此，后续将进一步探索基于原始数据表示 的特征学习方法, 尤其是面向复杂模型的结构识 别, 对称性分析的学习方法.

\section{参考文献(References):}

[1] Besl P J, McKay N D. A method for registration of 3-D shapes[J]. IEEE Transactions on Pattern Analysis and Machine Intelligence, 1992, 14(2): 239-256

[2] Rogers M, Graham J, et al. Robust and accurate registration of 2-D electrophoresis gels using point-matching[J]. IEEE Transactions on Image Processing, 2007, 16(3): 624-635

[3] Bronstein A M, Bronstein M M, Kimmel R. Efficient computation of isometry-invariant distances between surfaces[J]. SIAM Journal on Scientific Computing, 2006, 28(5): 1812-1836

[4] Osada R, Funkhouser T, Chazelle B, et al. Shape distributions[J]. ACM Transactions on Graphics, 2002, 21(4): 807-832

[5] Ovsjanikov M, Bronstein A M, Bronstein M M, et al. Shape Google: a computer vision approach to isometry invariant shape retrieval[C] //Proceedings of the 12th IEEE International Conference on Computer Vision Workshops. Los Alamitos: IEEE Computer Society Press, 2009: 320-327

[6] Kim V G, Lipman Y, Funkhouser T. Blended intrinsic maps[J]. ACM Transactions on Graphics, 2011, 30(4): Article No.79

[7] Sahillioğlu Y, Yemez Y. Scale normalization for isometric shape matching[J]. Computer Graphics Forum, 2012, 31(7): 2233-2240

[8] Barequet G, Sharir M. Partial surface and volume matching in three dimensions[J]. IEEE Transactions on Pattern Analysis and Machine Intelligence, 1997, 19(9): 929-948

[9] Shilane P, Funkhouser T. Selecting distinctive 3D shape descriptors for similarity retrieval[C] //Proceedings of the IEEE International Conference on Shape Modeling and Applications. Los Alamitos: IEEE Computer Society Press, 2006: 108-117

[10] Shilane P, Funkhouser T . Distinctive regions of 3D surfaces[J]. ACM Transactions on Graphics, 2007, 26(2): Article No.7

[11] Ferreira A, Marini S, Attene M, et al. Thesaurus based 3D object retrieval with part-in-whole matching[J]. International Journal of Computer Vision, 2010, 89(2-3): 327-347

[12] Tang K K, Song P, Chen X P. Signature of geometric centroids for 3D local shape description and partial shape matching $[\mathrm{C}] / /$ Proceedings of Asian Conference on Computer Vision. Heidelberg: Springer, 2017: 311-326

[13] Su H, Maji S, Kalogerakis E, et al. Multi-view convolutional neural networks for 3D shape recognition[OL]. [2020-06-22]. https://arxiv.org/abs/1505.00880

[14] Wang P S, Liu Y, Guo Y X, et al. O-CNN: octree-based convolutional neural networks for 3D shape analysis[J]. ACM Trans- actions on Graphics, 2017, 36(4): 72

[15] Qi C R, Yi L, Su H, et al. PointNet++: deep hierarchical feature learning on point sets in a metric space[OL]. [2020-06-22]. https://arxiv.org/abs/1706.02413

[16] Han Li, Piao Jingyu, Lan Pengyan, et al. 3D shape classification method for structural perceived depth learning[J]. Journal of Computer-Aided Design \& Computer Graphics, 2021, 33(1): 29-38

(韩丽, 朴京钜, 兰鹏燕, 等. 结构感知深度学习的三维形状 分类方法 [J]. 计算机辅助设计与图形学学报, 2021，33(1): 29-38)

[17] Lavoué G. Bag of words and local spectral descriptor for 3D partial shape retrieval[C] //Proceedings of the 4th Eurographics Conference on 3D Object Retrieval. New York: ACM Press, 2011: 41-48

[18] Han Z Z, Liu Z B, Vong C M, et al. BoSCC: bag of spatial context correlations for spatially enhanced $3 \mathrm{D}$ shape representation[J]. IEEE Transactions on Image Processing, 2017, 26(8): 3707-3720

[19] Liang Ye, Yu Jian, Liu Hongzhe. Study of BoF model based image representation[J]. Computer Science, 2014, 41(2): 36-44(in Chinese)

(梁华, 于剑, 刘宏哲. 基于 $\mathrm{BoF}$ 模型的图像表示方法研究 [J]. 计算机科学, 2014, 41(2): 36-44)

[20] Hilaga M, Shinagawa Y, Kohmura T, et al. Topology matching for fully automatic similarity estimation of 3D shapes[C] // Proceedings of the 28th Annual Conference on Computer Graphics and Interactive Techniques. New York: ACM Press, 2001: 203-212

[21] Sun J, Ovsjanikov M, Guibas L. A concise and provably informative multi-scale signature based on heat diffusion[J]. Computer Graphics Forum, 2009, 28(5): 1383-1392

[22] Sinha A, Bai J, Ramani K. Deep learning 3D shape surfaces using geometry images[C] //Proceedings of European Conference on Computer Vision. Heidelberg: Springer, 2016: 223-240

[23] Mémoli F, Sapiro G. A theoretical and computational framework for isometry invariant recognition of point cloud data[J]. Foundations of Computational Mathematics, 2005, 5(3): 313-347

[24] Han Li, Liu Shuning, Xu Shengsi, et al. Non-rigid 3D model classification algorithm based on adaptive sparse coding fusion[J]. Journal of Computer-Aided Design \& Computer Graphics, 2019, 31(11): 1898-1907(in Chinese) (韩丽, 刘书宁, 徐圣斯, 等. 自适应稀疏编码融合的非刚性 三维模型分类算法算法 [J]. 计算机辅助设计与图形学学报, 2019, 31(11): 1898-1907)

[25] Sahillioğlu Y, Yemez Y. Scale normalization for isometric shape matching[J]. Computer Graphics Forum, 2012, 31(7): 2233-2240

[26] Sahillioğlu Y, Yemez Y. Partial 3D correspondence from shape extremities[J]. Computer Graphics Forum, 2014, 33(6): 63-76

[27] Du G G, Yin C L, Zhou M Q, et al. Isometric 3D shape partial matching using GD-DNA[J]. Journal of Computer Science and Technology, 2018, 33(6): 1178-1191

[28] Litany O, Rodolà E, Bronstein A M, et al. Non-rigid puzzles[J]. Computer Graphics Forum, 2016, 35(5): 135-143 